\title{
Contribution of outer electron and inner - shell electron to proton energy loss in liquid water and DNA
}

\author{
Bashier Sabty Faris, Riaybd K.A. Al-ani \\ Al-Mustansiriyah University, College of Science, Department of Physics. \\ Baghdad-Iraq \\ E-mail address: Ahmed_naji_abd@yahoo.com
}

\begin{abstract}
Keywords: Ionizing radiation; Energy-loss function (ELF); Dielectric formalism; Drude-dielectric function; Partial Stopping Power Effective Charge (PSPEC); Generalized Oscillator Strength (GOS); Liquid water and DNA target
\end{abstract}

ABSTRACT. In the present work Drude-dielectric formalism has been used to calculate the effects of contribution of inner and outer-electron shell to energy-loss of protons liquid water $\boldsymbol{H}_{2} \mathrm{O}$ and $\boldsymbol{D N A}$. The results show that the incident protons with energy between $(\boldsymbol{T}=0.05,0.25,1,2,2.5$ $\mathbf{M e V}$ ) are very efficient in producing secondary electrons in dry $\boldsymbol{D N A}$, which are able to produce strand breaks and could be very effective for the biological damage of malignant cells. The PSPEC, Stopping power, average energy transfer, $\langle\Delta E\rangle$ and ionization energy have been calculated taking in the consideration the Sub-shells of each elements in $\boldsymbol{D N A}$ and $\boldsymbol{H}_{2} \boldsymbol{O}$, Fourier energy density $\boldsymbol{\rho}(\boldsymbol{q})$, Charge exchange and Screening effects (A). Good agreement achieved with the previous work [1].

\section{INTRODUCTION}

Studying the effect of outer electron and inner-shell electron to proton energy loss in $\boldsymbol{D} \boldsymbol{N} \boldsymbol{A}$ and Liquid water as a target, proton is one of the types of ionizing radiation and the study of the interaction of ionizing radiation (X-rays, electrons, positrons, protons or heavier ions) with living tissues has a paramount importance in cancer therapy, since the amount of energy deposited by the ionizing radiation to tumor cells will determine the outcome of the treatment [2].

When a proton (One of the types of ionizing radiations) moving inside living tissues, such as :liquid water $\mathrm{H}_{2} \mathrm{O}$ and $\mathrm{DNA}$ which are the most relevant biological materials ,we'll get energy distribution of the electronic excitations in this process, hence the focus will be on these materials as targets. Several models have been proposed to describe the electronic response of the targets [3].

It is worth mentioning the dielectric formulation $\in(\boldsymbol{k}, \boldsymbol{\omega})$ which was obtained by Lindhard (1954) for a free electron gas had been the basis of many applications and had become one of the most used methods to describe the interaction of swift ions and other charged particles with matter. The use of this formalism is to study the energy loss of charged particles was introduced by Fermi (1940) in his classical treatment of the density effect in the stopping power of relativistic particles in dense media. Secondary electrons which produced by ionizations induced in the living tissues by a fast projectile also contribute to the cellular damage. These electrons are able to travel and produce further ionizations in the $\boldsymbol{D N A}$, eventually leading to the cellular death [4]. The lethal efficiency of each secondary electron depends on its energy [5, 6], therefore it is very important to have information about the energy and number of the electrons generated by the projectile in the target.

The dielectric formalism $\boldsymbol{\varepsilon}(\boldsymbol{k}, \boldsymbol{\omega})$ as a function for each the wave vector $(\boldsymbol{k})$ and frequency $(\boldsymbol{\omega})$, dielectric function has significant consequences for physical properties of solids. The dielectric formulation had become one of the most used methods since it describes the interaction of swift ions and other charged particles with material. We can use this formalism for studying the energy loss of charged particles was introduced by Fermi (1940) since then, this has been a subject of continuous and growing interest. This formula (dielectric function) which obtained by Lindhard (1954) for a free electron gas has a lot of importance to the content of the important guaranteed by the imaginary part that can be brokered account the energy lost of the different levels therefor, it has 
been the basis of many applications. The imaginary part of dielectric function or dielectric constant of valence electrons in material has known as the mutant energy and momentum variable (k) where unilateral dispersion occurs is flexible, dielectric function is used and the imaginary part depending on the type of polarization caused by falling on the target ion.

\section{THEORY}

\section{II.1 Partial Stopping Power Effective Charge (PSPEC)}

In 1900 Drude-model of electrical conduction was proposed [7] by Paul Drude to explain the transport properties of electrons in matters (especially metals). This model is an application of kinetic theory, considers that the microscopic behaviour of electrons in a solid may be treated classically and looks much like a pinball machine, with a sea of constantly jittering electrons bouncing and re-bouncing off heavier. Drude-model based on the assumption that the matter can be described as a gas of free electrons is a well-known model for predicting the optical properties of good metals [8].

Drude-model $\boldsymbol{E L F s}$ can be written as follow equation:-

$$
\operatorname{Im}\left[\frac{-1}{\epsilon(\mathrm{k}, \omega)}\right]=\sum_{\mathrm{i}} \frac{\mathrm{A}_{\mathrm{i}} \gamma_{\mathrm{i}}}{\left(\mathrm{w}-\mathrm{w}_{\mathrm{i}}\right)^{2}+(\mathrm{w} \gamma)^{2}}
$$

Where:- $\boldsymbol{A}_{i, \boldsymbol{D}}, \boldsymbol{\omega}_{\boldsymbol{i}}, \boldsymbol{\gamma}_{\boldsymbol{i}}$ : are the intensity, position, width, and the subscript $\boldsymbol{D}$ stands for Drude .

Later this model was extended to finite values of $\mathbf{k}$ namely the extended-Drude model [9], the improved extended-Drude model [10,11].

The presence of the imaginary part in dielectric function is very important and that what it's content of detailed information on the energy levels of the valence electrons in materials, called exchange loss or (energy loss), can also be brokered account energy ground level [12, 13].

With the dielectric-function formalism, the energy loss cross-section of a singly charged projectile with velocity $\boldsymbol{v}$ can be expressed as follow [14]:-

$$
\mathrm{S}_{(\mathrm{q})}=\frac{2}{\mathrm{~N} \pi \mathrm{v}^{2}} \int_{0}^{\infty} \frac{\mathrm{dk}}{\mathrm{k}}|\rho(\mathrm{k})|^{2} \int_{0}^{\mathrm{kv}} \mathrm{d} \omega \omega \operatorname{Im}\left[\frac{-1}{\epsilon(\mathrm{k}, \omega)}\right]
$$

Where $\boldsymbol{N}:$ is the atomic density of the medium, $\boldsymbol{p}(\boldsymbol{q}):$ is Fourier transform charge density.

$$
\rho(\mathrm{k})=\mathrm{z}_{1} \frac{\mathrm{q}+(\mathrm{k} \Lambda)^{2}}{1+(\mathrm{k} \Lambda)^{2}}
$$

In eq.(2) the imaginary part $\operatorname{Im}\left[\frac{-1}{\epsilon(\boldsymbol{k}, \boldsymbol{\omega})}\right]$ will be divided into two parts

$$
\operatorname{Im}\left[\frac{-1}{\epsilon(k, \omega)}\right]=\operatorname{Im}\left[\frac{-1}{\epsilon(k, \omega)}\right]_{\text {outer }}+\operatorname{Im}\left[\frac{-1}{\epsilon(k, \omega)}\right]_{\text {inner }}
$$

The first term is for the outer electron excitations which is calculated by using Drude-model energy loss function, while the second part for the inner shell electron excitation which is calculated by using the generalized oscillator strengths (GOS) of the constituent atoms.

The Partial Stopping Power Effective Charge (PSPEC), $\zeta(\boldsymbol{q})$ fraction of energy loss is given by :-

$$
\zeta=\left[\frac{\mathrm{s}_{\mathrm{q}}}{\mathrm{s}_{\mathrm{q}=1}}\right]^{1 / 2}
$$


Where $\left(\boldsymbol{S}_{\boldsymbol{q}=1}\right)$ which represents the energy loss for the bare nucleus of the projectile.

$$
S_{(q=1)}=\frac{2}{N \pi v^{2}} \int_{0}^{\infty} \frac{d k}{k} \int_{0}^{k v} d \omega \omega \operatorname{Im}\left[\frac{-1}{\epsilon(k, \omega)}\right]
$$

The dielectric-function of Outer-shell is given by $\boldsymbol{E q} .1$

The stopping power of charged particle $\boldsymbol{S}(\boldsymbol{q})$ of individual element given in $D N A\left(C_{20} H_{27} N_{7} O_{13} P_{2}\right)$ has been calculated numerically and used to find the (PSPEC) of $D N A$ by using Bragg's additivity-rule :-

$$
S_{D N A}(q)=20 S_{C}(q)+27 S_{H}(q)+7 S_{N}(q)+13 S_{o}(q)+2 S_{P}(q)
$$

For water $\mathrm{H}_{2} \mathrm{O}$ :-

$$
S_{H 2 O}(q)=2 S_{H}(q)+S_{o}(q)
$$

Where $\boldsymbol{S}_{\boldsymbol{i}}(\boldsymbol{q})$ refers to the (PSPEC) of each elements in $\boldsymbol{D N A}$ in eq.(7) and for each elements in liquid water in eq.(8).

\section{II.2 Dipole Oscillator strength}

In (1984) J. Oddershede and J. R. Sabin derived relation to find the $\boldsymbol{G O S}$ for each valance electron and sub-shell [15]. The cross-section according to the stopping number $\boldsymbol{L}(\boldsymbol{v})$ can be given as :-

$$
S(v)=\frac{4 \pi e^{4} Z_{1}^{2} Z_{2}}{m v^{2}} L(v)
$$

Taking the summation over $\boldsymbol{j t h}$. Shell as follow:-

$$
\begin{aligned}
& L(v)=\sum_{j} L_{j}(v) \\
& S(v)=\sum_{j} S_{j}(v)
\end{aligned}
$$

Where the summation is over the atomic shells :-

$$
L_{j}(v)=\pi \int_{0}^{\infty} P_{k}\left(v_{2}\right) v_{2} d v_{2} \times \int_{\left|v-v_{2}\right|}^{v+v_{2}} L_{j}^{o}(v)\left[1+\frac{v^{2}}{v^{\prime 2}}-\frac{v_{2}^{2}}{v^{\prime 2}}\right] d v^{\prime}
$$

Where: $\boldsymbol{P}_{\boldsymbol{k}}$ : represents the density.

The velocity distribution of the scatters has been chosen as the $\boldsymbol{k t h}$ shell electron velocity distribution, and by taking into consideration the normalization we get :-

$$
4 \pi \int_{0}^{\infty} P_{k}\left(v_{2}\right) v_{2}^{2} d v_{2}=1
$$

By using Bethe-formula for $\boldsymbol{L}_{\boldsymbol{j}}^{\mathbf{0}}(\boldsymbol{v})$ we get :

$$
L_{j}^{0}(v)=\frac{\omega_{j}}{Z_{2}} \ln \left(\frac{2 m v^{2}}{I_{j}}\right) \theta\left(v-\alpha_{j}\right)
$$

And $\alpha_{j}=\left(I_{j} / 2 m\right)^{1 / 2}$, where $:-\omega_{j}:$ is the orbital weight factor. $\left(v-\alpha_{j}\right):$ is a Heaviside function limits the range of integration to the range of validity of the Bethe-formula, therefore For carrying out the decomposition of $\boldsymbol{L}(\boldsymbol{v})$ into orbital terms as eq.(10), the mean excitation energy (I) must also spilt into orbital terms

$$
\ln I=\frac{1}{Z_{2}} \sum_{j} \omega_{j} \ln I_{j}
$$

Where $\omega_{j}$ : in a neutral atom, satisfy

$$
\begin{aligned}
& \sum_{j} \omega_{j}=Z_{2} \\
& S(0)=\sum_{j} n_{j} f_{j} \equiv \sum_{j} S_{j}(0)
\end{aligned}
$$




\section{Where :-}

$\boldsymbol{f}_{\boldsymbol{j}}:$ represents the orbital oscillator strength, so the total oscillator strength is :-

$$
\begin{aligned}
& S_{j}=n_{j} f_{j} \\
& n_{j} f_{j}=2 \omega_{j}-n_{j} \\
& S_{j}=2 \omega_{j}-n_{j} \\
& \omega_{j}=\frac{n_{j}+S_{j}}{2} \\
& S_{\text {total }}=\sum_{j} f_{j} S_{j}=\sum_{j}\left(\frac{2 \omega_{j}-1}{n_{j}}\right) S_{j}
\end{aligned}
$$

Where :- $\boldsymbol{n}_{\boldsymbol{j}}$ : is no. of electron in each jth. shell.

\section{Results and Discussion}

Inner and outer-shells have different binding energies and the binding energy of inner-shells are a larger than outer-shells. Different responses of the target's valence-band and inner-shell electrons to the perturbation which caused by inelastic collisions that happen between the incident charged particle and target's electrons can be described by using energy loss function (ELF).

In which the outer electron excitations of the targets (DNA and $\mathrm{H} 2 \mathrm{O}$ and) are calculated for Drude-model energy loss function. The accurately matches the available experimental data for liquid water and $\boldsymbol{D N A}$ obtained from optical measurements, while the inner-shell electron excitation are modeled by the dipole oscillator strengths of the constituent atoms, then apply Bragg's rules In order to get all the data of the compound completely.

A program (Bashier-H-DNA.for) written in FORTRAN 90 has been used by using software Computer Visual Fortran V6.6 for executing the program [Bashier,2015].

Now, show the results of the relation between inner-stopping power $\boldsymbol{S}_{\boldsymbol{s h l}}$ in a.u with charge fraction (q) for each element in $\mathrm{DNA}$ and $\mathrm{H}_{2} \mathrm{O}$ with $(\mathrm{T}=0.05,0.25,1,2,2.5 \mathrm{MeV} / u)$.

$S_{D N A}(q)=20 S_{C}(q)+27 S_{H}(q)+7 S_{N}(q)+13 S_{o}(q)+2 S_{P}(q)$

And for liquid water, $H_{2} \mathrm{O}:-\mathbf{S}_{\mathrm{H}_{2} \mathrm{O}}(\mathbf{q})=2 S_{\mathrm{H}}(\mathbf{q})+S_{0}(\mathbf{q})$

For DNA $\left(\mathrm{C}_{20} \mathrm{H}_{27} \mathrm{~N}_{7} \mathrm{O}_{13} \mathrm{P}_{2}\right)$ :-

Figs [(1), (2), (3), (4), (5)] show the variation of stopping power of sub-shell $\boldsymbol{S}_{\boldsymbol{s h l}}$ in $\boldsymbol{a} . \boldsymbol{u}$. with charge-fraction $(\boldsymbol{q})$ for Carbon-element, Hydrogen-element, Nitrogen-element, Oxygen-element, phosphorus-element, respectively with $(T=0.05,0.25,1,2,2.5 \mathrm{MeV} / \mathrm{u})$.

Stopping power of Sub-shells at $\boldsymbol{q}=\mathbf{0}$, for $\boldsymbol{C}, \boldsymbol{H}, \boldsymbol{N}, \boldsymbol{O}$ and $\boldsymbol{P}$ elements is equal to zero and for five incident proton-energies, $T=0.05,0.25,1,2$ and $2.5 \mathrm{MeV}$ as shown in figs.(1-5).

While at $\boldsymbol{q}=\mathbf{1}$ stopping power is vary as follow :-

1- For $C$-element, $Z=6, C\left(1 S^{2} 2 S^{2} 2 P^{2}\right)$

$$
S_{P}\left(1 S^{2}\right)>S_{P}\left(2 P^{2}\right)>S_{P}\left(2 S^{2}\right)
$$

2- For $N$-element, $Z=7, N\left(1 S^{2} 2 S^{2} 2 P^{3}\right)$

$$
S_{P}\left(2 P^{3}\right)>S_{P}\left(1 S^{2}\right)>S_{P}\left(2 S^{2}\right)
$$

3- For $O$-element, $Z=8, O\left(1 S^{2} 2 S^{2} 2 P^{4}\right)$

$$
S_{P}\left(2 P^{4}\right)>S_{P}\left(1 S^{2}\right)>S_{P}\left(2 S^{2}\right)
$$


4-For $P$-element, $Z=15, P\left(1 S^{2} 2 S^{2} 2 P^{6} 3 S^{2} 3 P^{3}\right)$

$$
S_{P}\left(2 P^{6}\right)>S_{P}\left(1 S^{2}\right)>S_{P}\left(3 P^{3}\right)>S_{P}\left(2 S^{2}\right)>S_{P}\left(3 S^{2}\right)
$$

At last the stopping power of Sub-shells is inversely proportional with incident protons energy $\boldsymbol{T}$ $(\mathbf{M e V})$. The most contribution shell to stopping power is $\boldsymbol{P}$-shell for elements $\boldsymbol{N}, \boldsymbol{O}$ and $\boldsymbol{P}$. For carbon $(C), \boldsymbol{I S}$-shell is the most contribution to the stopping power.

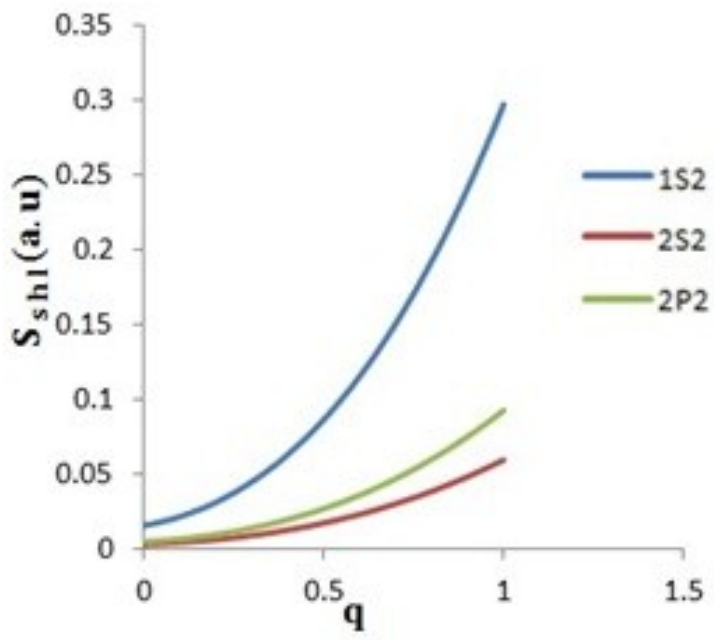

(a)

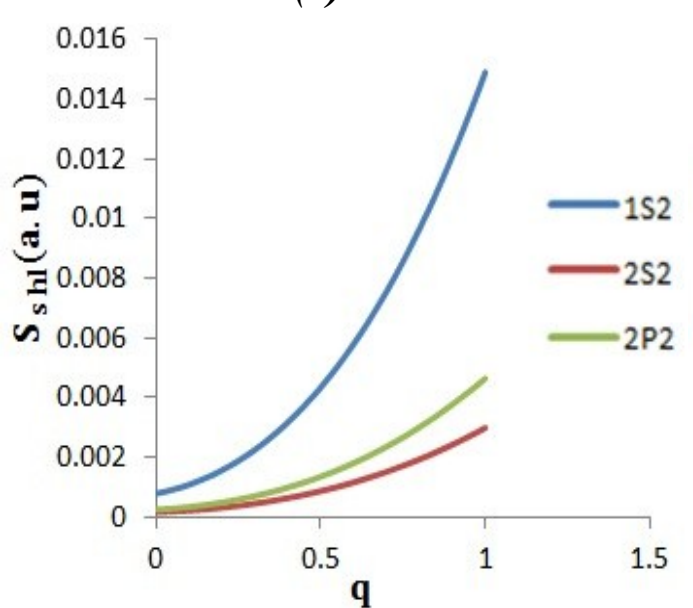

(c)

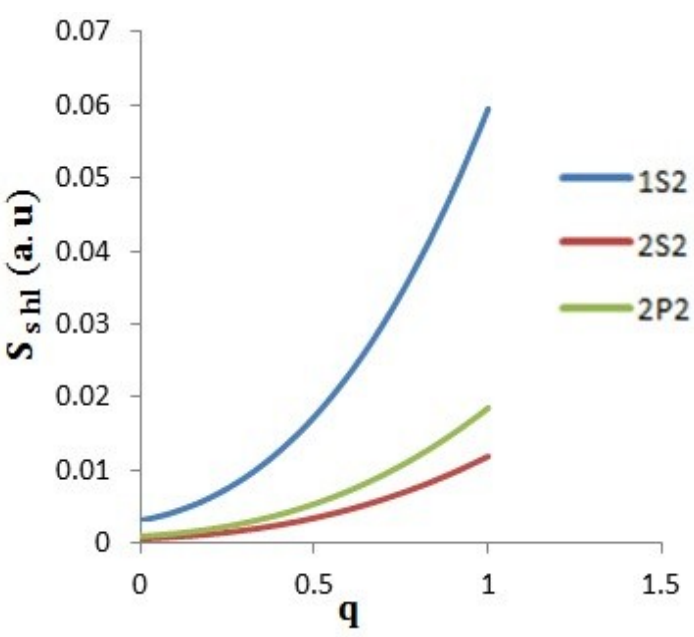

(b)

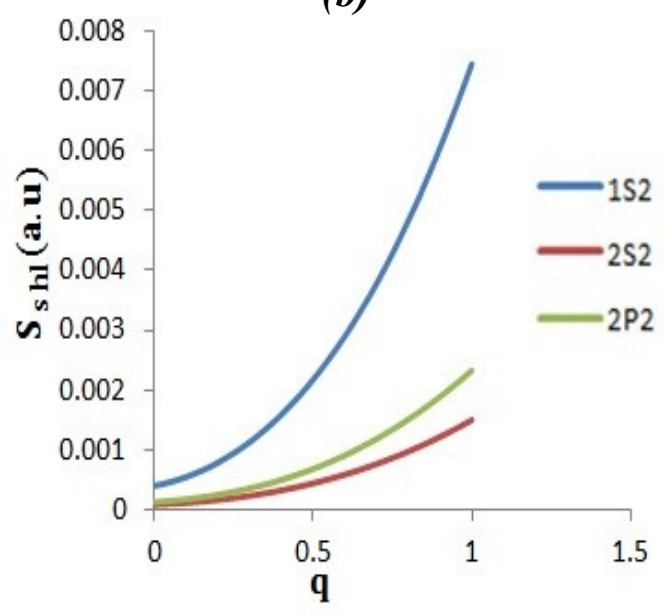

(d)

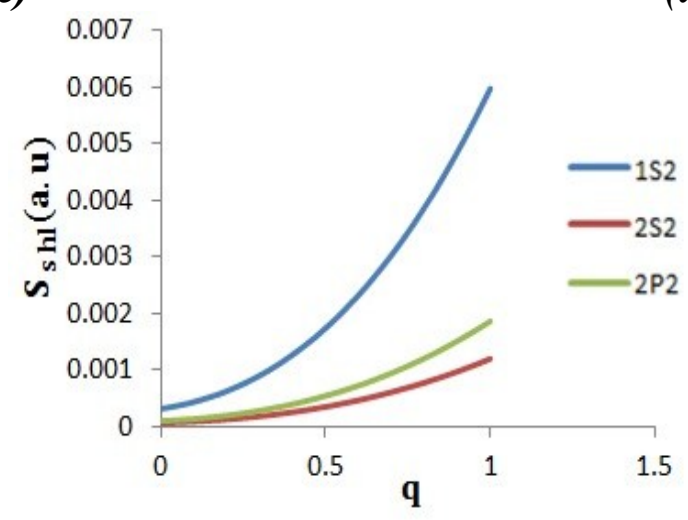

(e)

Fig.(1) The variation of stopping power of sub-shell $S_{\text {shl }}$ in a.u. with charge fraction (q) for $C$ element, with $(T=0.05,0.25,1,2,2.5 \mathrm{MeV} / \mathrm{u})$ 

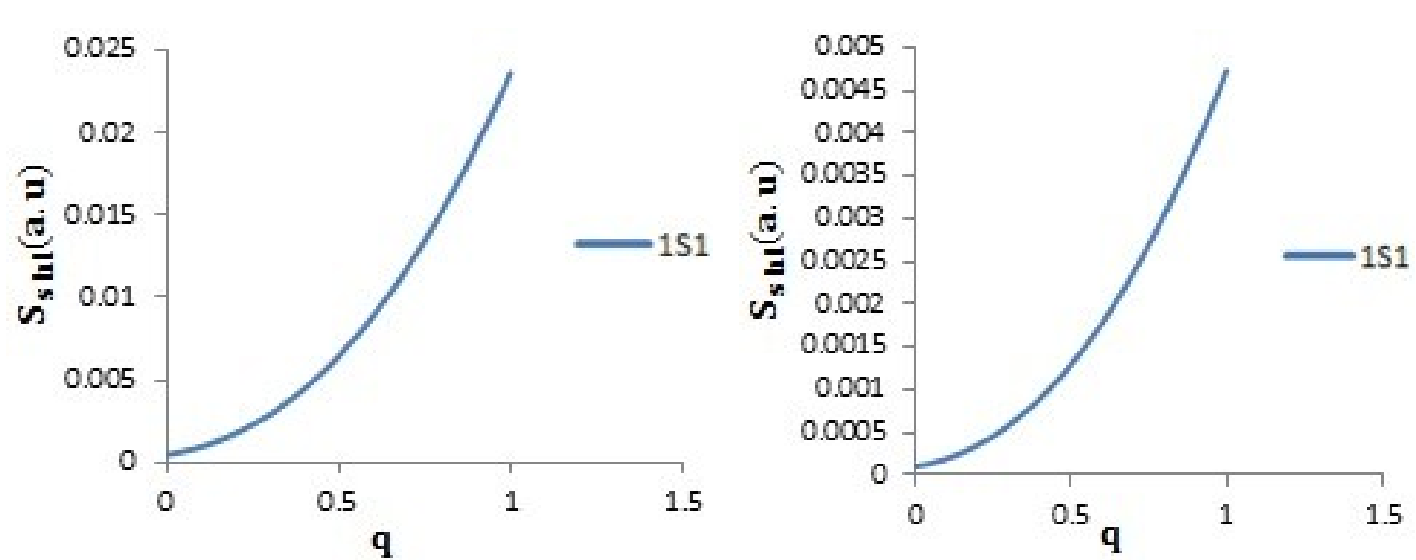

(a)

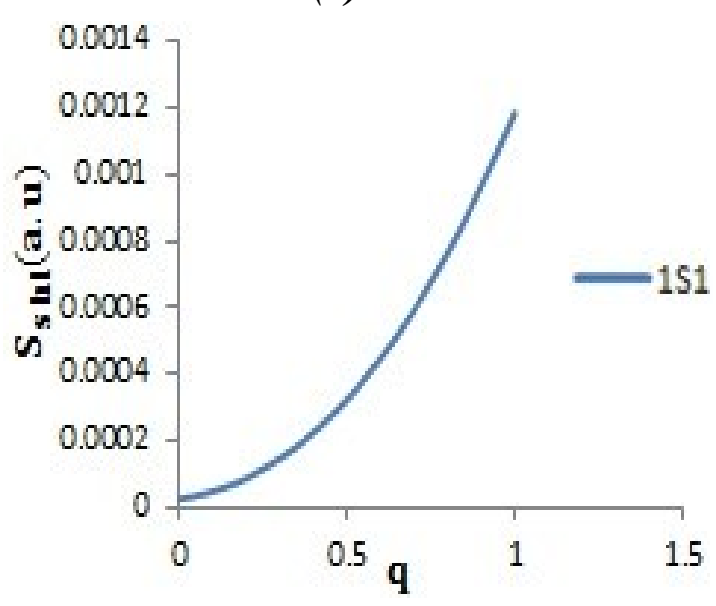

(b

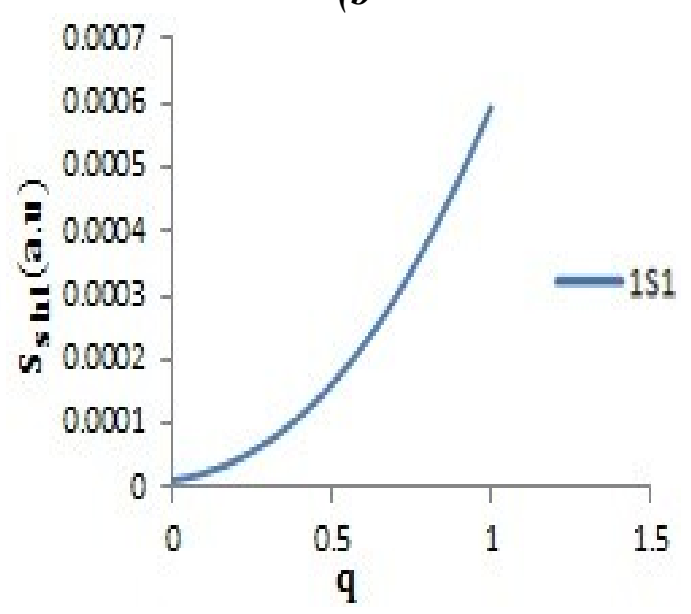

(c)

(d)

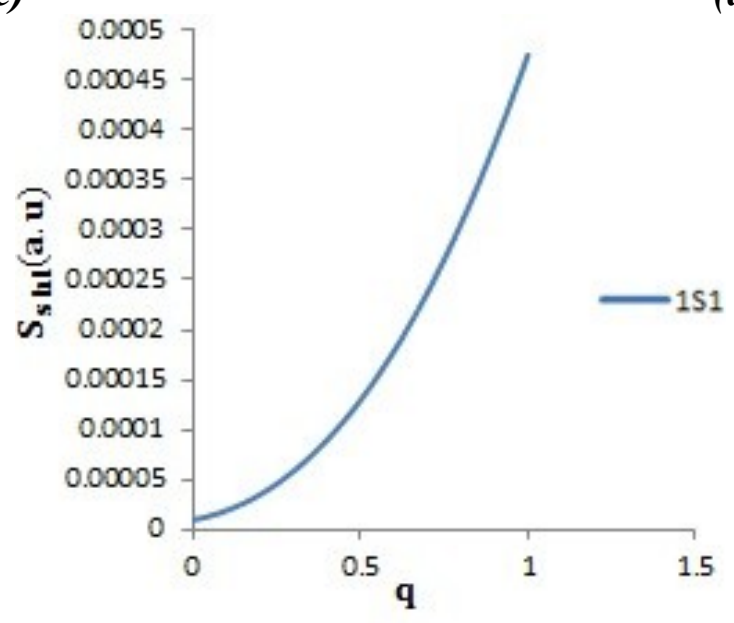

(e)

Fig.(2) The variation of stopping power of sub-shell $S_{\text {shl }}$ in a.u. with charge fraction (q) for $H$ element, with $(T=0.05,0.25,1,2,2.5 \mathrm{MeV} / \mathrm{u})$ 




(a)

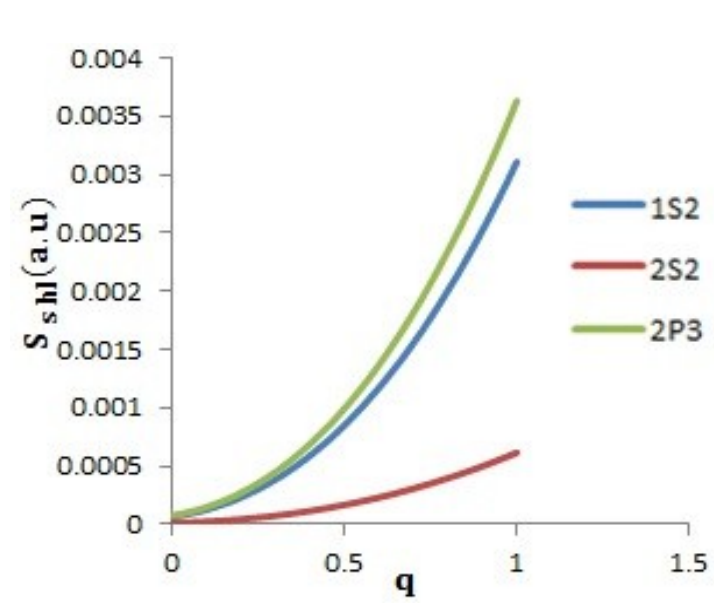

(c)

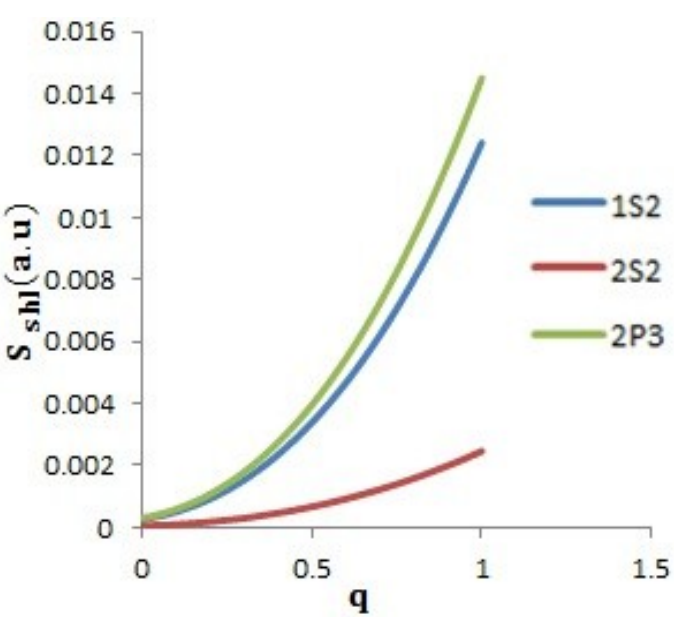

(b)

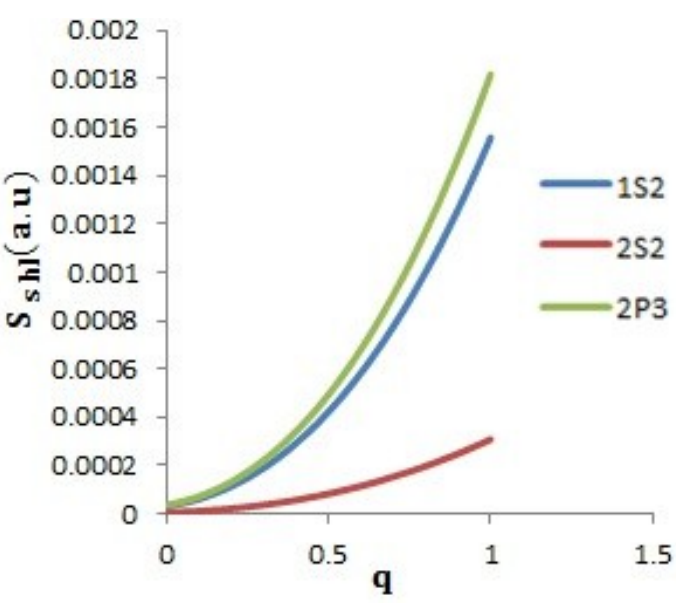

(d)

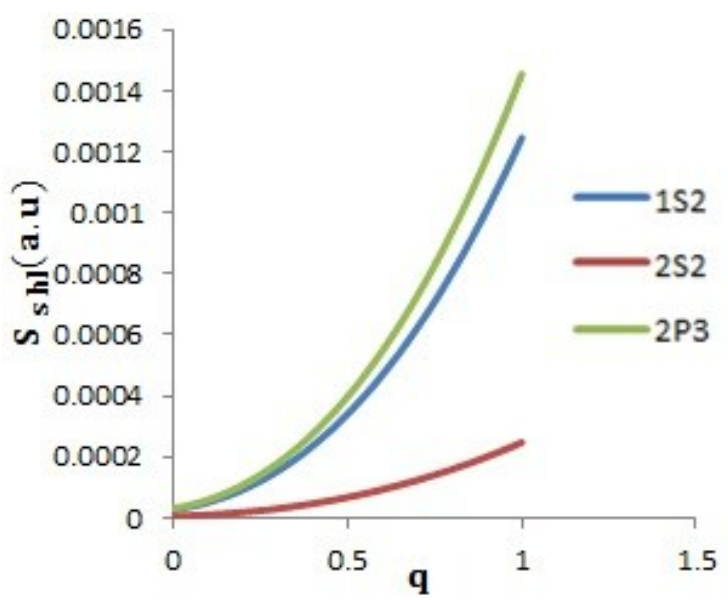

(e)

Fig.(3) The variation of stopping power of sub-shell $S_{\text {shl }}$ in a.u. with charge fraction (q) for $N$ element, with $(\mathrm{T}=0.05,0.25,1,2,2.5 \mathrm{MeV} / \mathrm{u})$ 


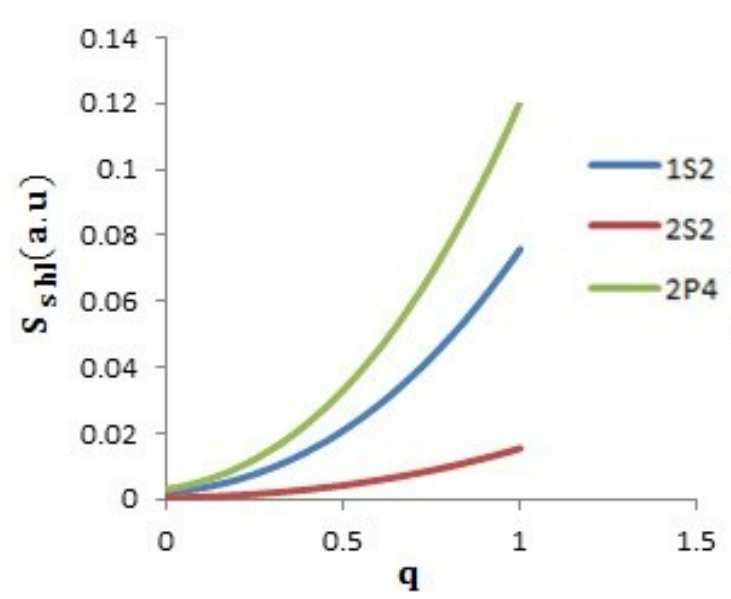

(a)

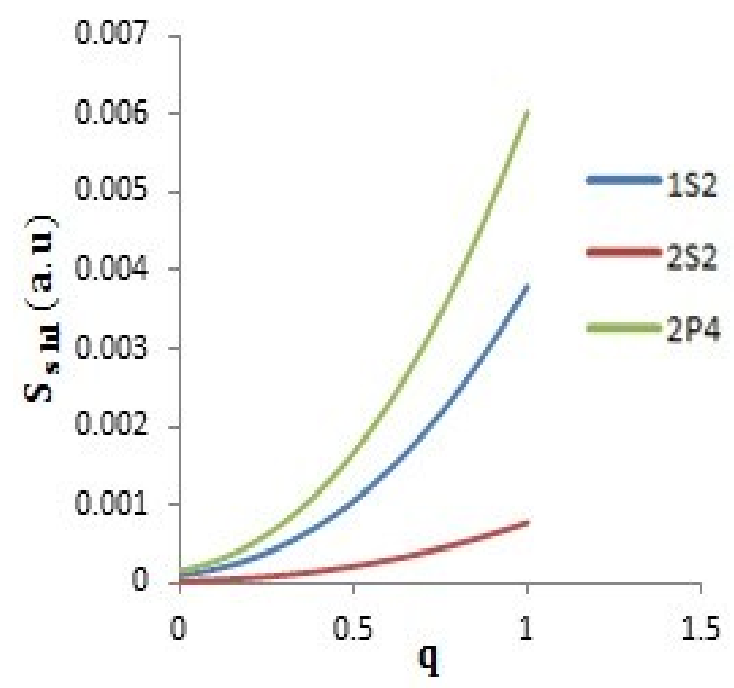

(c)

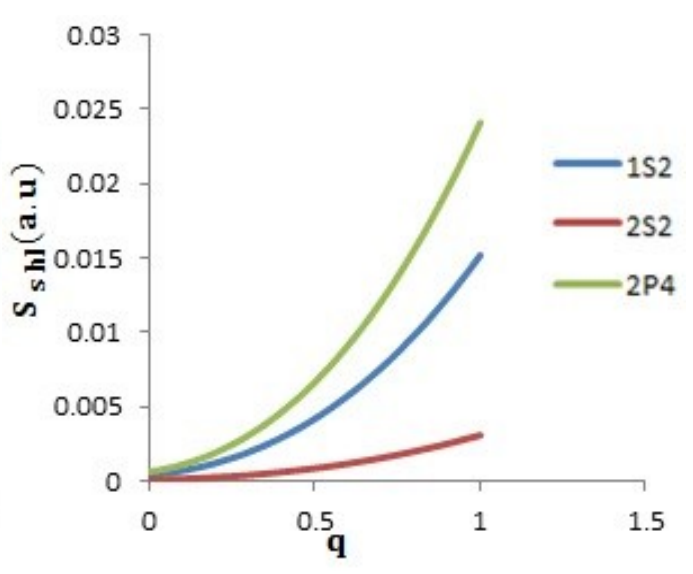

(b)

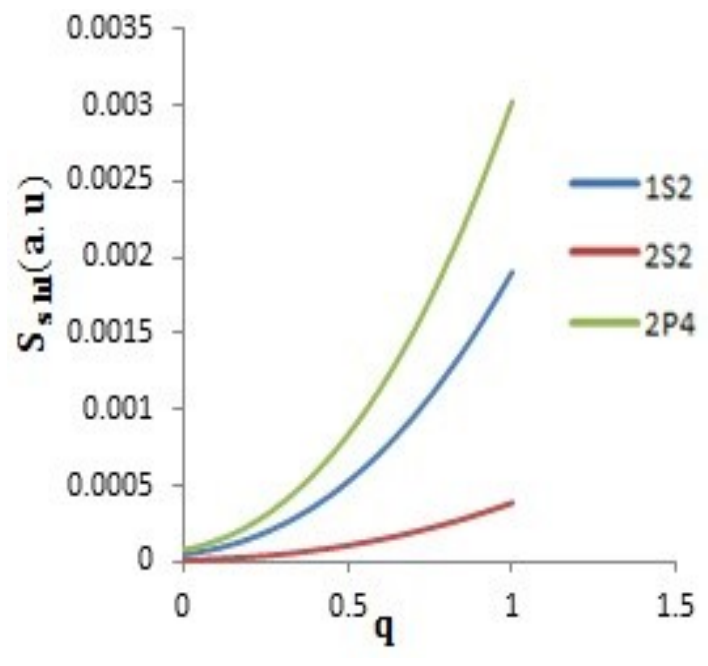

(d)

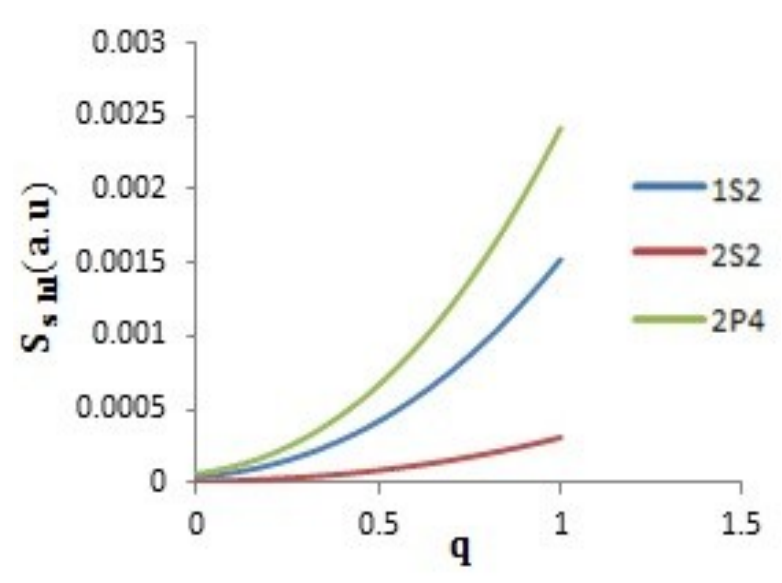

(e)

Fig.(4) The variation of stopping power of sub-shell $S_{\text {shl }}$ in a.u. with charge fraction (q) for $O$ element, with $(\mathrm{T}=0.05,0.25,1,2,2.5 \mathrm{MeV} / \mathrm{u})$ 


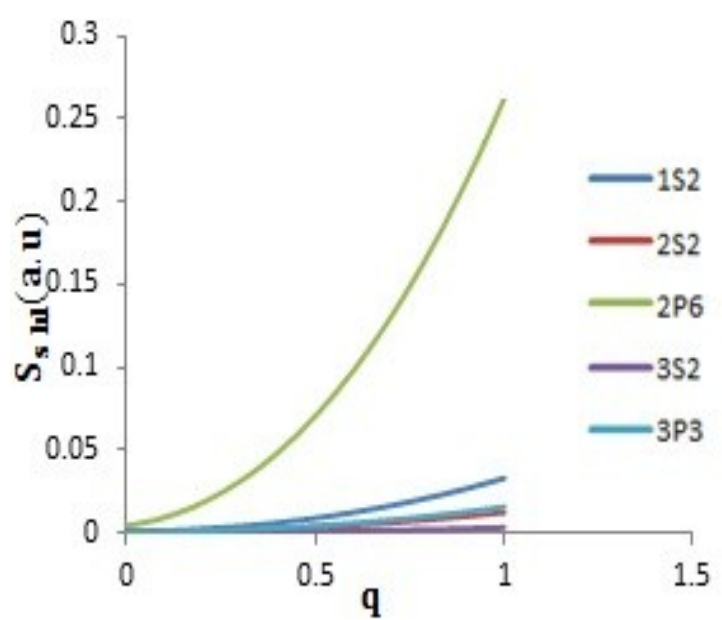

(a)

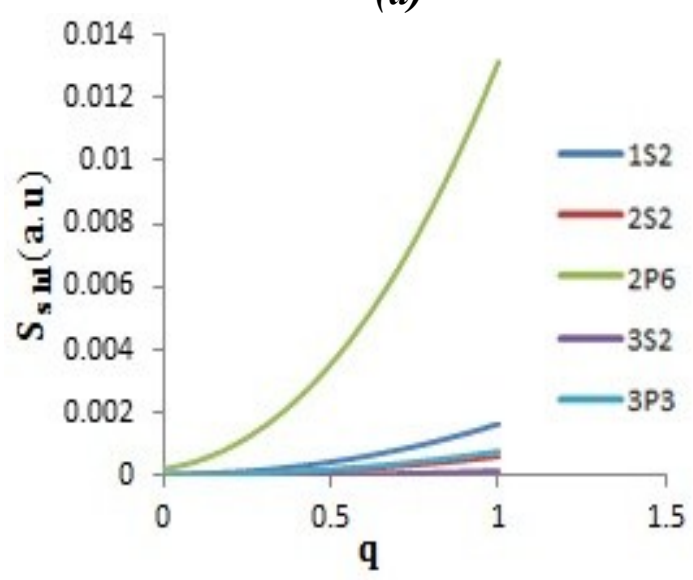

(c)

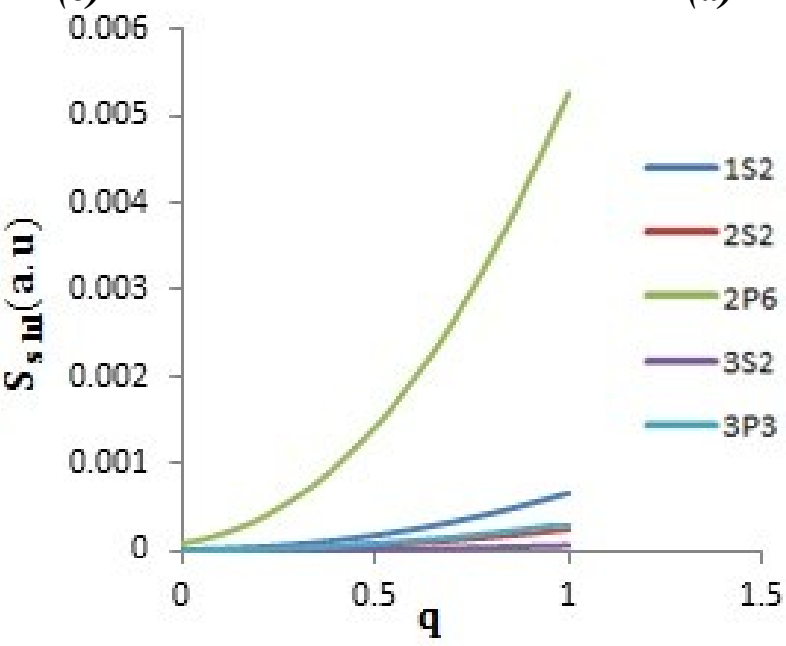

(e)

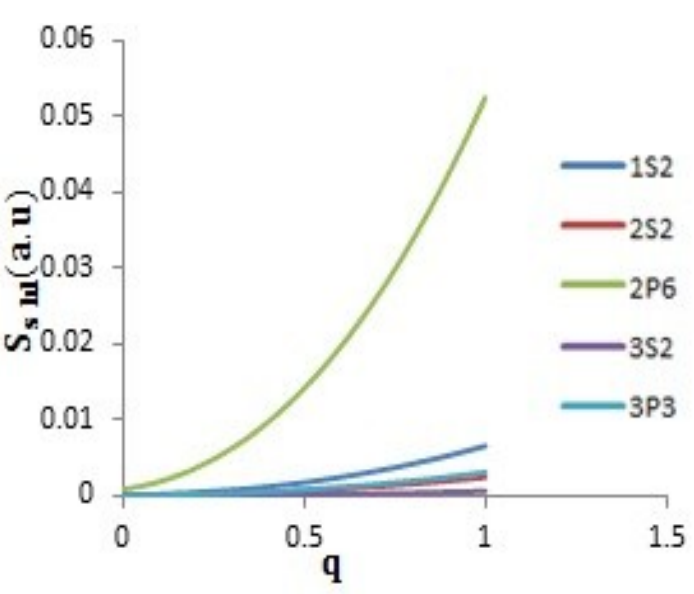

(b)

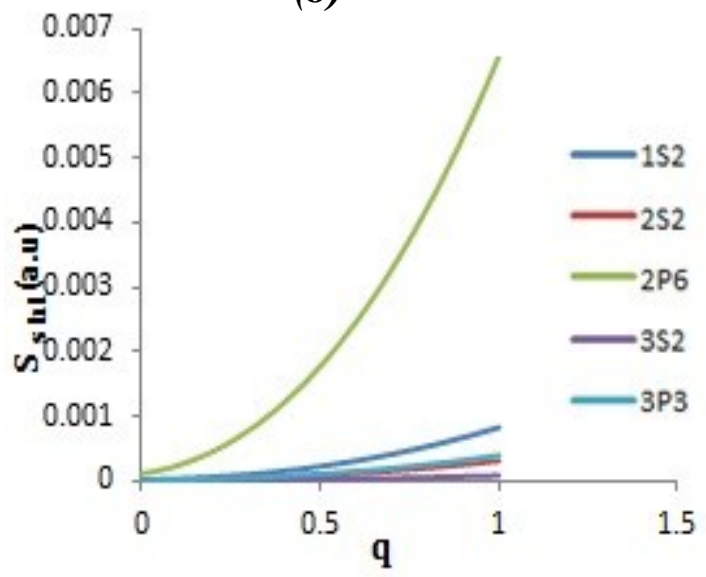

(d)

Fig.(5) The variation of stopping power of sub-shell $S_{\text {shl }}$ in a.u. with charge fraction (q) for $P$ element, with $(T=0.05,0.25,1,2,2.5 \mathrm{MeV} / \mathrm{u})$

\section{Conclusions}

In the present work the Drude-dielectric formalism have been used to calculate the variation effects of stopping power of sub-shell in $\boldsymbol{a} . \boldsymbol{u}$ with charge fraction $\boldsymbol{q}$ in two targets of high biological interest, namely, $\boldsymbol{D N A}$ and $\boldsymbol{H}_{\mathbf{2}} \boldsymbol{O}$, has been studied taking into consideration the Sub-shells of each elements in $\boldsymbol{D N A}$ and $\boldsymbol{H}_{\mathbf{2}} \boldsymbol{O}$. The main points in the present work are listed below :-

1- When charged particles such as :- (protons) pass through a targets (dry DNA and liquid water), these particles (protons) energy transmitted gradually to the target atoms, mainly transmitted by 
inelastic collisions with electrons target atoms and then the result of this process occurs ionizations and excitations for atoms of the target.

2- After the loss of energy for incident charged particles (i.e protons) gradually at inner-part of the target and the speed is decreasing to become close to the speed of the electrons orbit $\boldsymbol{K}$ to the targets (liquid water and dry $\mathbf{D N A}$ ), as a result gets ionizations and excitations of the target atoms, then these incident particles (Protons) turning to neutral atoms as hydrogen $\boldsymbol{H}$.

3- The variation of stopping power of Sub-shell $\boldsymbol{S}_{\boldsymbol{s h} \boldsymbol{l}}$ in $\boldsymbol{a} . \boldsymbol{u}$ units with charge fraction $\boldsymbol{q}$ is inversely proportional with incident protons energy $\boldsymbol{T}(\mathbf{M e V})$, and the most contribution shell to stopping power is $\boldsymbol{P}$-shell for elements $\boldsymbol{N}, \boldsymbol{O}$ and $\boldsymbol{P}$. For carbon $(\boldsymbol{C}), \boldsymbol{I S}$-shell is the most contribution to the stopping power as shown in figs.[(1)-(5)].

\section{References}

[1] Isabel Abril, Rafael Garcia-Molina, Cristian D. Denton, Ioanna Kyriakou, and Dimitris Emfietzoglou, Energy Loss of Hydrogen and Helium-Ion Beams in DNA Calculations Based on a Realistic Energy Loss Function of the Target, RADIATION RESEARCH,175,247-255,2011.

[2] [H. Breuer and B.J. Smit ,(2000) ; E.B. Podgorsak ,(2006)].

[3] Isabel Abril,Cristian D.Denton,pablo de vera, Loanna kyriakou, Dimitris Emfietzoglou, Rafael Garcia-Molina, Effect of the Bethe surface description on the electronic excitations induced by energetic proton beams in liquid water and DNA Nucl. Instr. and Meth. In Phys. Res. B, Vol.268, 1763-1767, 2010.

[4] Cai Z. and Coutier P., (2005), J. Phys. Chem. B 109,(2005)4796.

[5] Boudaïffa B., Cloutier P., Hunting D., Huels M.A., Sanche L., (2000) Science 287,1658.

[6] Sanche L., (2002), Radiat. Prot. Dosim. 99,57.

[7] Drude, Paul (1900),"Zur Elektronentheorie der metalle". Annalen der Physik (306 (3): $566 ; 308$ (11):369).

[8] Rakic, A. D., Djurisic, A. B., Elazar, J. M. and Majewski, M. L., (1998),Optical properties of

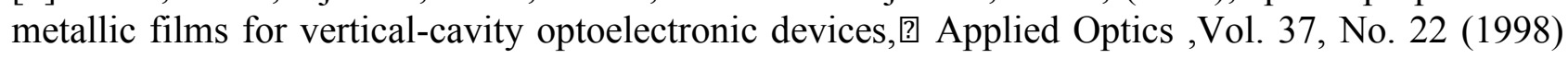
pp. 5271-5283.

[9] R.H. Ritchie, A. Howie, Phil. Mag. 36 (1977) 463 .

[10] D. Emfietzoglou, F.A. Cucinotta, H. Nikjoo, Radiat. Res. 164 (2005) 202 .

[11] D. Emfietzoglou, R. Garcia-Molina, I. Kyriakou, I. Abril, H. Nikjoo, Phys. Med Biol. 54 (2009) 3451 .

[12] E. Fermi, Z. Phys. 29, 315(1924); Phys. Rev. 56, 1242(1939); 57, 485(1940).

[13] Burns G.,(1985),"Solid state physics" 1st Edition, Academic Press, Inc.(1985).

[14] Echenique P.M., Nagy I., and Arnau A. (1989)International Journal of Quantum Chemistry. Quantum Chemistry Symposium 23(1989) 521.

[15] Sabin, J.R. and Oddershde, J. (1984)."Electronic Stopping Powers for Low Projectile Velocities," Phys. Rev. A29, No.4, 1757. 